\title{
A Scalable and Lightweight QoS Monitoring Technique Combining Passive and Active Approaches
}

\section{- On the Mathematical Formulation of CoMPACT Monitor -}

\author{
Masaki Aida*, Naoto Miyoshi ${ }^{\dagger}$ and Keisuke Ishibashi* \\ *NTT Information Sharing Platform Laboratories \\ NTT Corporation \\ Email: \{aida.masaki,ishibashi.keisuke\}@lab.ntt.co.jp \\ ${ }^{\dagger}$ Department of Mathematical and Computing Sciences \\ Tokyo Institute of Technology \\ Email: miyoshi@is.titech.ac.jp
}

\begin{abstract}
To make a scalable and lightweight QoS monitoring system, we have proposed a new QoS monitoring technique, Change-of-Measure based Passive/Active Monitoring (CoMPACT Monitor), which is based on change-of-measure framework and is an active measurement transformed by using passively monitored data. This technique enables us to measure detailed QoS information for individual users, applications, and organizations, in a scalable and lightweight manner. In this paper, we present the mathematical foundation of CoMPACT Monitor. In addition, we show its characteristics through simulations in terms of typical implementation issues for inferring the delay distributions. The results show that CoMPACT Monitor gives accurate QoS estimations with only a small amount of extra traffic for active measurement.
\end{abstract}

\section{INTRODUCTION}

The Internet has been growing rapidly with respect to the number of users and the amount of traffic and has been recognized as an important infrastructure for information in social and business use. So, although the initial and main issue of the Internet has been its connectivity and transmission capacity, attention has recently been paid to its quality too. The traffic conveyed by the Internet is generated by a wide variety of applications, which have different characteristics and different quality requirements. Thus, Quality of Service (QoS) and performance measurements are crucial in controlling and managing QoS and provisioning networks. However, it is difficult or expensive to measure QoS and performance statistics of each flow directly.

Recently, many monitoring tools have been developed to monitor network performance [1], [2], [3], [20] and their measurement results have also been reported [10], [21], [23]. In general, conventional monitoring schemes to measure QoS and the performance of networks are classified into two types: active and passive monitoring. Unfortunately, both types have drawbacks. They are briefly summarized as follows.

a) Active measurement monitors QoS and the performance of a network by sending probe packets and monitoring them
[6], [7], [22]. There are various active methods to measure network performance such as available bandwidth [16], delay, loss, and to estimate their link-by-link performance [12]. They monitor the QoS/performance of the probe-packet stream to determine the QoS/performance of the user/network indirectly. This means that we implicitly assume that the QoS/performance of a user/networks is the same as the values measured from active probe packets. These active monitoring schemes have the following problems.

- If we use a probe-packet stream that simulates an actual user traffic:

- The probe-packet stream causes non-negligible amount of extra traffic on the networks and it affects QoS/performance of users' traffic and

- The QoS/performance obtained from the probe packets is not equal to that without the influence of the probe-packet stream.

- If we use short probe packets and send them in certain intervals, like ping:

- The extra traffic may be negligible, but the QoS/performance obtained from the probe packets is not equal to the QoS/performance experienced by users, in general.

Let us add some explanation about the last case. Since the time for sending probe packets is independent of the users' behaviors, QoS/performance measured by the active monitoring scheme generally differs from the actual QoS/performance that users experience. If and only if we can assume that active monitoring measures the time average of network performance and that the user traffic is Poissonian, then the performance experienced by the users and the actively-measured performance will be the same. This well-known property is called PASTA [25]. It is known, however, that current Internet traffic exhibits bursty properties and does not generally have Poisson arrivals [19]. In that case, an average user experiences worse 
performance than the time-average performance measured by active monitoring.

b) Passive measurement is mainly used to monitor traffic volume but can measure network performance as well. Passive monitoring is categorized into two types: two-point monitoring and one-point monitoring.

- Two-point monitoring requires two monitoring devices deployed at ingress and egress points in a network. The devices sequentially take packet data, and network performance parameters such as delay and loss can be calculated by comparing the data of the corresponding packets taken at each point. If we apply the two-point monitoring to measure QoS/performance:

- All devices should have synchronized timing.

- The two-point monitoring requires identifying each packet at the two devices by its header and/or contents. Since this identification process is hard when the packet volume is huge, as in a large-scale network, the two-point monitoring does not have scalablility.

- To identify the monitored packets, we should collect all the packet data. This process requires nonnegligible bandwidth.

- One-point monitoring uses the TCP acknowledgment mechanism. When a TCP-sink receives a packet from a TCP-source, it transmits an acknowledgement for the packet [24]. Thus, by monitoring the packet-ack pair at a point in the network, we can measure the round-trip delay between the point and the sink. The packet loss can also be detected in this way. However, if we apply one-point monitoring, measurement is restricted to TCP flows.

Our approach, Change-of-Measure based Passive/Active Monitoring (CoMPACT Monitor), is different from the above methods. It combines both active and passive monitoring using easy-to-measure methods. It is based on change-ofmeasure framework and is an active measurement transformed by using passively monitored data. CoMPACT Monitor can estimate not only the mixed QoS/performance experienced by users but also the actual QoS/performance for individual users, organizations, and applications. In addition, CoMPACT Monitor is scalable and lightweight, where the scalability in this paper means the monitoring system does not become complex even if the volume and/or number of target flows (e.g., user flows) that are sharing the common path increases.

We have proposed the concept of CoMPACT Monitor in [5], and have investigated the characteristics of its simple implementation in [15]. However, our previous works do not present its mathematical foundation. So, the condition, in which CoMPACT Monitor works well, has not been clarified. In this paper, we present the mathematical foundation of CoMPACT Monitor and show its characteristics with respect to typical implementation issues for inferring the delay distributions.

The rest of the paper is organized as follows. In section II, we briefly summarize the concept of CoMPACT Monitor and related works that combine passive and active methods. In section III, we present mathematical formulations of the framework for CoMPACT Monitor. In section IV, we investigate implementations of CoMPACT Monitor. To demonstrate the features of CoMPACT Monitor, section V shows examples of delay distribution measurements for individual users via simulation. In addition, we show the characteristics of CoMPACT Monitor with respect to implementation issues. Finally, we conclude the paper in section VI.

\section{CONCEPT OF COMPACT MONITOR}

\section{A. Concept}

CoMPACT Monitor is a scalable and lightweight monitoring technique that enables us to estimate detailed characteristics of performance for individual users, organizations, and applications. It combines simple measurements of both active and passive types by change-of-measure framework.

We can recognize that the measurement of QoS statistics fundamentally corresponds to integrals. This is because it is an accumulation of some quantities according to a certain rule. Our technique enables us to obtain statistics of the measurement objective not from the integral describing a direct measurement of the objective, but from other integral that is easy to measure. These integrals are in different forms but their values are the same.

Let $X_{k}$ be the measurement objective, e.g., the delay for user $k$ 's packets, whose distribution function is $F_{k}$. The distribution of $X_{k}$ is obtained as follows:

$$
\begin{aligned}
\operatorname{Pr}\left(X_{k}>a\right) & =\int 1_{\{x>a\}} \mathrm{d} F_{k}(x) \\
& =\mathrm{E}_{F_{k}}\left[1_{\left\{X_{k}>a\right\}}\right],
\end{aligned}
$$

where $\mathrm{E}_{F_{k}}$ denotes the expectation with respect to $F_{k}$ and $1_{\{\cdot\}}$ denotes the indicator function.

Suppose we have a situation in which it is difficult to measure $X_{k}$ directly, and an estimate of its distribution cannot be obtained with (1). Let $V(t)$ be the network performance at time $t$ and $X_{k}$ be the value of $V(t)$ measured at a certain time. Also, let $Y$ be the value of $V(t)$ measured independently of $X_{k}$ and let the distribution function of $Y$ be $G$. Then, $\operatorname{Pr}\left(X_{k}>a\right)$ in (1) is obtained, under a certain condition, as

$$
\begin{aligned}
\operatorname{Pr}\left(X_{k}>a\right) & =\int 1_{\{y>a\}} \frac{\mathrm{d} F_{k}(y)}{\mathrm{d} G(y)} \mathrm{d} G(y) \\
& =\mathrm{E}_{G}\left[1_{\{Y>a\}} \frac{\mathrm{d} F_{k}(Y)}{\mathrm{d} G(Y)}\right],
\end{aligned}
$$

where $\mathrm{E}_{G}$ denotes the expectation with respect to $G$ and $\mathrm{d} F_{k} / \mathrm{d} G$ denotes the likelihood ratio of $F_{k}$ with respect to $G$.

If $Y$ is easy to measure and we can derive $\mathrm{d} F_{k}(Y) / \mathrm{d} G(Y)$, then the estimator of the distribution of $X_{k}$ can be derived with the measurement values of $Y$. The fundamental concept of CoMPACT Monitor is as follows: The estimation of the distribution $\operatorname{Pr}\left(X_{k}>a\right)$ from direct measurements of $X_{k}$ 
is difficult. However, if the values $Y$ and $\mathrm{d} F_{k}(Y) / \mathrm{d} G(Y)$ are easily obtained by respectively using active and passive monitoring, then we can easily estimate the distribution $\operatorname{Pr}\left(X_{k}>a\right)$ from (2). The likelihood ratio can be obtained through simple counting of user packets.

In addition, (2) means that if we have $\mathrm{d} F_{k}(Y) / \mathrm{d} G(Y)$ for each class $k$ traffic (user, application, organization, and their combination specified by $k ; k=1,2, \ldots$ ), we can simultaneously obtain the individual QoS for each class $k$ traffic sharing a common path from the common active measurement of $Y$.

We can expect CoMPACT Monitor to have the following advantages:

- Negligible extra traffic for active probe packets: Since the extra traffic for active probe packets can be small, user traffic is little affected.

- Simplification of passive monitoring devices: To measure the delay distribution, the conventional passive monitoring requires two-point monitoring. However, passive monitoring in CoMPACT Monitor only requires packet counting. Since that is one-point monitoring, passive monitoring devices are simplified (Fig. 1).

- Simultaneous estimation of QoS for individual packet streams:

By determining the individual likelihood ratio for each packet stream sharing a common path, we can obtain QoS parameters for an individual packet stream from the common active monitored sequence. The likelihood ratio is easily determined by counting the number of packets in the packet stream filtered by any class we want to measure.

- Protocol independence:

CoMPACT Monitor is applicable to non-TCP protocols as well.

Here, we add some explanations to the scalability of CoMPACT Monitor. The target flows measurable by the common active measurement of $Y$ are restricted to the flows sharing the same physical and logical path. When we measure the target flows in different paths, it is necessary to use different active probe packet sequences (of course, this is the same feature as conventional active measurements). Therefore, if all the target flows share the same path, we can measure all the flows by using a single active probe packet sequence and the monitoring system is not complicated with respect to increase of the number of target flows (as well as increase of the volume of target flows). CoMPACT Monitor has this type of the scalability. In addition, although different active probe packet sequences are required for measuring the target flows in different paths, configuration of passive monitoring devices is not always complicated. If the different paths include the common link, we can reduce the number of passive monitoring devices by fixing the passive monitoring device at the common link (Fig. 2).

\section{B. Related Work}

Lindh has proposed a new QoS monitoring technique that combines passive and active ways [17], [18]. In this technique,
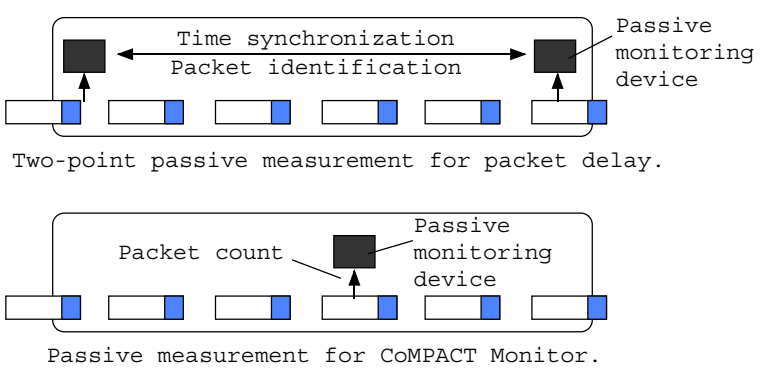

Fig. 1. Simplification of passive monitoring for CoMPACT Monitor.

a router sends active probe packets at regular intervals such that the number of target user packets passing through the router becomes a predefined fixed value. The passive monitoring device is used to count the number of packets. Since the density of active probe packets inserted into the network is proportional to the density of the target user packet stream, this technique also can measure $\mathrm{QoS}$ for a packet stream, e.g., the QoS experienced by a user. However, this technique has the following drawbacks:

- This technique inserts many active probe packets when there are many packets in the target packet flow (e.g., user flow). This means that the number of active probe packets tends to increase when the network is congested. Thus, active probe packets will affect the QoS of users' traffic.

- If we need to measure the individual QoS for each user, this technique inserts different active probe packet sequences corresponding to the users. Thus, this technique does not have scalability with respect to an increase in the number of target packet flows.

However, in CoMPACT Monitor, the extra traffic for active probe packets is independent of user traffic, and an individual QoS can be derived with the common active probe packet sequence even when the number of target packet flows, in the same path, increase.

\section{Mathematical Formulation of CoMPACT MONITOR}

This section presents the mathematical formulation of CoMPACT monitor in order to clarify the conditions in which it works well. Since Internet traffic has a wide variety of characteristics, we present the mathematical formulation for nonstationary user traffic. This formulation is also applicable to stationary user traffic.

In the framework of CoMPACT Monitor, there are many user packets in the network compared with active probe packets. Thus, we consider a fluid approximation of user traffic; that is, user packets are approximated as a fluid, while the arrivals of active probe packets are modeled as a point process.

Suppose that a path in the network is shared by $K(\geq$ 1) users. Let $A_{k}(t), k=1, \ldots, K$, denote the cumulative amount of fluid transmitted by user $k$ observed during $(0, t]$, 


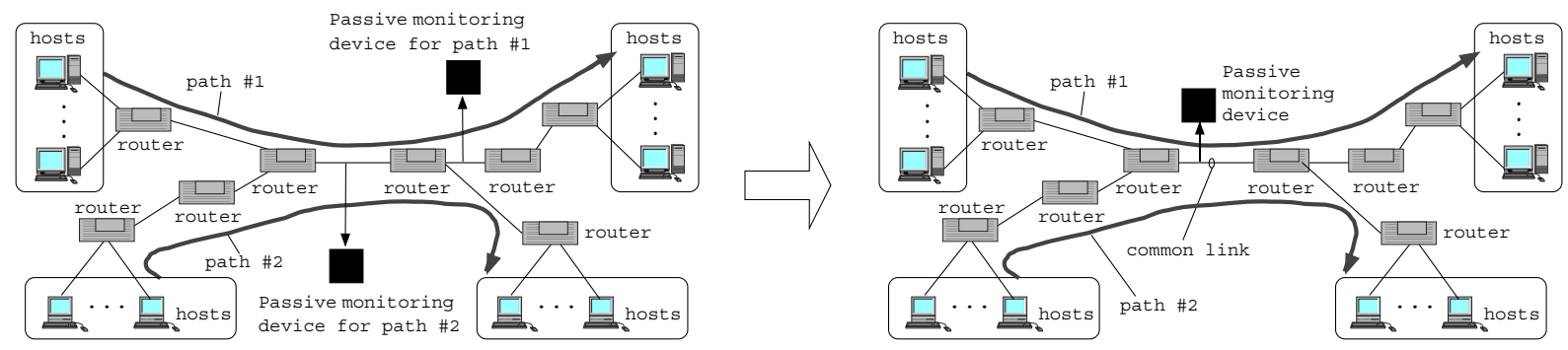

Fig. 2. Simplification of the configuration of passive monitoring devices.

where each $\left\{A_{k}(t)\right\}_{t>0}$ is a deterministic, real-valued and nondecreasing process satisfying $A_{k}(0)=0$. We assume that $\liminf _{t \rightarrow \infty} A_{k}(t) / t>0$ and that $\left\{A_{k}(t)\right\}_{t \geq 0}$ is absolutely continuous with density $\left\{a_{k}(t)\right\}_{t>0}$; that is, $A_{k}(t)=$ $\int_{0}^{t} a_{k}(s) \mathrm{d} s, t \geq 0$, where we also assume that $\left\{a_{k}(t)\right\}_{t \geq 0}$ is right-continuous with left-limits and is bounded on $t \geq 0$. Let $V(t)$ denote the virtual delay in the path at time $t$, where $\{V(t)\}_{t \geq 0}$ is a deterministic, real-valued, and nonnegative process. We assume that $\{V(t)\}_{t \geq 0}$ is right-continuous with leftlimits in $t \geq 0$. The deterministic process $\left\{\left(V(t), a_{k}(t) ; k=\right.\right.$ $1, \ldots, K)\}_{t \geq 0}$ is considered as a sample path extracted from the corresponding stochastic process, where we assume neither stationarity nor ergodicity. Note that $\{V(t)\}_{t \geq 0}$ is influenced not only by $\left\{a_{k}(t) ; k=1, \ldots, K\right\}_{t \geq 0}$ but also other flows that share all/a part of the path of $\left\{a_{k}(t) ; k=1, \ldots, K\right\}_{t \geq 0}$.

Define $A_{k}^{-1}(x)=\inf \left\{t \geq 0: A_{k}(t) \geq x\right\}, k=1, \ldots, K$, for $x \geq 0$. Note that $A_{k}^{-1}(x)$ represents the time at which the cumulative fluid transmitted by user $k$ reaches level $x$. More intuitively, we can interpret $A_{k}^{-1}(x)$ as the arrival time of the "fluid molecule (packet) labeled $x$ " transmitted by user $k$. The delay $W_{k}(x)$ of the "fluid molecule $x$ " of user $k$ is then represented by $W_{k}(x)=V\left(A_{k}^{-1}(x)\right)$ for $x \geq 0$. Therefore, for any $y>0$ and any $k=1, \ldots, K$, the empirical average network delay distribution for the fluid of user $k$ over the amount $y$ of fluid is given by

$$
\begin{aligned}
\pi_{k, y}(C) \\
=\frac{1}{y} \int_{0}^{y} 1_{\left\{W_{k}(x) \in C\right\}} \mathrm{d} x \\
=\frac{1}{y} \int_{0}^{y} 1_{\left\{V\left(A_{k}^{-1}(x)\right) \in C\right\}} \mathrm{d} x \\
=\frac{A_{k}^{-1}(y)}{y} \frac{1}{A_{k}^{-1}(y)} \\
\quad \times \int_{0}^{A_{k}^{-1}(y)} 1_{\{V(s) \in C\}} a_{k}(s) \mathrm{d} s, \quad C \in \mathfrak{B}\left(\mathbb{R}_{+}\right) .
\end{aligned}
$$

Thus, since $A_{k}^{-1}(y) \rightarrow \infty$ as $y \rightarrow \infty$ due to the bounded property of $\left\{a_{k}(t)\right\}_{t \geq 0}$, if the limits

$$
\begin{gathered}
\lim _{t \rightarrow \infty} \frac{1}{t} \int_{0}^{t} 1_{\{V(s) \in C\}} a_{k}(s) \mathrm{d} s \\
\lim _{y \rightarrow \infty} \frac{y}{A_{k}^{-1}(y)}=\lim _{t \rightarrow \infty} \frac{A_{k}(t)}{t}=\lim _{t \rightarrow \infty} \frac{1}{t} \int_{0}^{t} a_{k}(s) \mathrm{d} s
\end{gathered}
$$

exist, we can see that there is a long-term average delay distribution for the fluid of user $k$ and it is given by

$$
\pi_{k}(C)=\lim _{y \rightarrow \infty} \pi_{k, y}(C), \quad C \in \mathfrak{B}\left(\mathbb{R}_{+}\right),
$$

where $\liminf _{t \rightarrow \infty} A_{k}(t) / t>0$ is ensured by the assumption.

Now, consider estimating the long-term average delay distribution $\pi_{k}(C), C \in \mathfrak{B}\left(\mathbb{R}_{+}\right)$, by monitoring the network at random sampling epochs. Let $\{N(t)\}_{t \geq 0}$ denote a simple counting process representing the monitoring epochs of the network and let $\left\{T_{n}\right\}_{n \geq 1}$ be the corresponding point sequence; that is, $T_{n}=\inf \{t \geq 0: N(t) \geq n\}$ for $n=1,2, \ldots$ We assume that $N$ has stationary and ergodic increments with respect to a probability measure $\mathrm{P}$ and also has the positive and finite intensity $\lambda_{N}=\mathrm{E}[N(1)]$, where $\mathrm{E}$ denotes the expectation with respect to $\mathrm{P}$. Then, we have the following:

Theorem 1 If, for some $k \in\{1, \ldots, K\}$ and some $C \in$ $\mathfrak{B}\left(\mathbb{R}_{+}\right)$, the following hold with constants $\alpha_{k}(C)$ and $\alpha_{k}$,

$$
\begin{aligned}
\lim _{m \rightarrow \infty} \frac{1}{m} \sum_{n=1}^{m} 1_{\left\{V\left(T_{n}\right) \in C\right\}} a_{k}\left(T_{n}\right) & =\alpha_{k}(C), \quad \text { P-a.s., } \\
\lim _{m \rightarrow \infty} \frac{1}{m} \sum_{n=1}^{m} a_{k}\left(T_{n}\right) & =\alpha_{k}, \quad \text { P-a.s., }
\end{aligned}
$$

then limits (4) and (5) also exist for such $k$ and $C$, and we have

$$
\begin{aligned}
\pi_{k}(C) & =\frac{\alpha_{k}(C)}{\alpha_{k}} \\
& =\lim _{m \rightarrow \infty} \frac{\sum_{n=1}^{m} 1_{\left\{V\left(T_{n}\right) \in C\right\}} a_{k}\left(T_{n}\right)}{\sum_{n=1}^{m} a_{k}\left(T_{n}\right)}, \quad \text { P-a.s. }
\end{aligned}
$$

Proof: We show that limit (4) exists and coincides with $\alpha_{k}(C)$ in (7) along similar lines to the proof of Theorem 3.1 in [14]. Since $\left\{\left(V(t), a_{k}(t)\right)\right\}_{t \geq 0}$ is a deterministic process, we have

$$
\begin{aligned}
& \mathrm{E}\left[\frac{1}{t} \sum_{n=1}^{N(t)} 1_{\left\{V\left(T_{n}\right) \in C\right\}} a_{k}\left(T_{n}\right)\right] \\
& =\mathrm{E}\left[\frac{1}{t} \int_{0}^{t} 1_{\{V(s) \in C\}} a_{k}(s) \mathrm{d} N(s)\right] \\
& =\frac{1}{t} \int_{0}^{t} 1_{\{V(s) \in C\}} a_{k}(s) \lambda_{N} \mathrm{~d} s,
\end{aligned}
$$


where we use the relation $\mathrm{E}[N(t)]=\lambda_{N} t$ due to the stationarity of $N$. Since $\left\{a_{k}(t)\right\}_{t \geq 0}$ is bounded, for the inside of the expectation in the left-hand side above, we have

$$
\frac{1}{t} \sum_{n=1}^{N(t)} 1_{\left\{V\left(T_{n}\right) \in C\right\}} a_{k}\left(T_{n}\right) \leq a_{k}^{\text {sup }} \frac{N(t)}{t},
$$

where $a_{k}^{\text {sup }}=\sup _{t>0}\left\{a_{k}(t)\right\}$ and $\mathrm{E}[N(t) / t]=\lambda_{N}<\infty$. Thus, applying the dominated convergence theorem to (10), we obtain

$$
\begin{aligned}
& \mathrm{E}\left[\lim _{t \rightarrow \infty} \frac{1}{t} \sum_{n=1}^{N(t)} 1_{\left\{V\left(T_{n}\right) \in C\right\}} a_{k}\left(T_{n}\right)\right] \\
& =\lim _{t \rightarrow \infty} \frac{\lambda_{N}}{t} \int_{0}^{t} 1_{\{V(s) \in C\}} a_{k}(s) \mathrm{d} s .
\end{aligned}
$$

For the limit on the left-hand side, we have by the condition of the theorem and the ergodicity of $N$ :

$$
\begin{aligned}
& \lim _{t \rightarrow \infty} \frac{1}{t} \sum_{n=1}^{N(t)} 1_{\left\{V\left(T_{n}\right) \in C\right\}} a_{k}\left(T_{n}\right) \\
& =\lim _{t \rightarrow \infty} \frac{N(t)}{t} \frac{1}{N(t)} \sum_{n=1}^{N(t)} 1_{\left\{V\left(T_{n}\right) \in C\right\}} a_{k}\left(T_{n}\right) \\
& =\lambda_{N} \alpha_{k}(C), \quad \text { P-a.s. }
\end{aligned}
$$

so limit (4) exists and coincides with $\alpha_{k}(C)$. The existence of limit (5) and its coincidence with $\alpha_{k}$ in (8) are immediate by replacing $C$ with $\mathbb{R}_{+}$. We then have result (9) from (3) and (6).

Theorem 1 shows that the long-term average delay distribution for the fluid of user $k$ is estimated through $m$-times monitoring of the network by

$$
Z_{k, m}(C ; N)=\frac{\sum_{n=1}^{m} 1_{\left\{V\left(T_{n}\right) \in C\right\}} a_{k}\left(T_{n}\right)}{\sum_{n=1}^{m} a_{k}\left(T_{n}\right)},
$$

which is indeed strongly consistent in the sense that

$$
\lim _{m \rightarrow \infty} Z_{k, m}(C ; N)=\pi_{k}(C), \quad \text { P-a.s., }
$$

provided that (7) and (8) hold.

Remark 1 In the above discussion, $\left\{\left(V(t), a_{k}(t) ; k=\right.\right.$ $1, \ldots, K)\}_{t \geq 0}$ is interpreted as a sample path extracted from the corresponding stochastic process, where we assume neither stationarity nor ergodicity of the stochastic process. Indeed, the value of $\left(\alpha_{k}(C), \alpha_{k}\right)$ in (7) and (8) may depend on the individual samples of $\left\{\left(V(t), a_{k}(t)\right)\right\}_{t \geq 0}$, while it can not depend on the sample of $\{N(t)\}_{t \geq 0}$ once a sample of $\left(\alpha_{k}(C), \alpha_{k}\right)$ is given. Furthermore, we can weaken the assumption of $\{N(t)\}_{t>0}$ such that it is asymptotically ergodic. Therefore, we can use as $\{N(t)\}_{t \geq 0}$ a non-delayed renewal process with a spread-out interarrival distribution (see [8]).

Remark 2 Also, once we assume that $\left\{\left(V(t), a_{k}(t)\right)\right\}_{t \geq 0}$ is stochastic and jointly ergodic with the sampling process $N$, the result (9) reduces to

$$
\mathrm{P}_{A_{k}}(V(0) \in C)=\frac{\mathrm{E}_{N}^{0}\left[1_{\{V(0) \in C\}} a_{k}(0)\right]}{\mathrm{E}_{N}^{0}\left[a_{k}(0)\right]},
$$

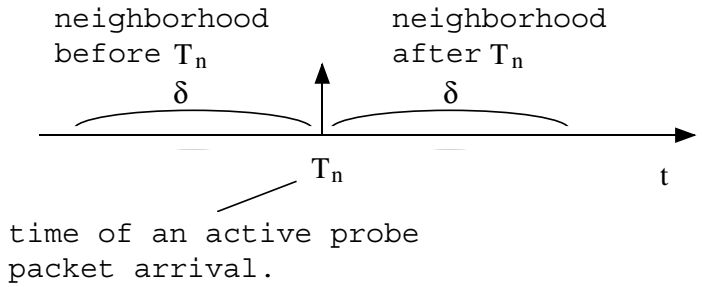

Fig. 3. Difference in implementations.

where $\mathrm{P}_{A_{k}}$ denotes the Palm probability defined by $\left(\mathrm{P}, A_{k}\right)$ and $\mathrm{E}_{N}^{0}$ denotes the expectation with respect to the Palm probability $\mathrm{P}_{N}^{0}$ defined by $(\mathrm{P}, N)$ (see [9] and [13]). In this case, we can replace the boundedness of $\left\{a_{k}(t)\right\}_{t>0}$ with $\mathrm{E}_{N}^{0}\left[a_{k}(0)\right]<\infty$. In this formula, we see that $a_{k}(0) / \mathrm{E}_{N}^{0}\left[a_{k}(0)\right]$ plays a role of the likelihood ratio $\mathrm{dP}_{A_{k}} / \mathrm{dP}_{N}^{0}$. This is just the origin of the name "Changeof-Measure-based" monitoring (see Section 2.1 and also [5], [15]).

Remark 3 In the above remarks, though $\left\{\left(V(t), a_{k}(t) ; k=\right.\right.$ $1, \ldots, K)\}_{t \geq 0}$ is considered stochastic, it is still assumed to be independent of the sampling process $N$. Now, consider the case where $\left(V(t), a_{k}(t)\right)$ depends on $\{N(s)\}_{s<t}$ for $t>0$. In this case, if $N$ is Poisson and $\left\{\left(V(t), a_{k}(t)\right)\right\}_{t>0}$ is jointly ergodic with $N$, we can verify the following by using the Poisson calculus [11] (see also [25]);

$$
\mathrm{P}_{A_{k}}(V(0) \in C)=\frac{\mathrm{E}_{N}^{0}\left[1_{\{V(0-) \in C\}} a_{k}(0-)\right]}{\mathrm{E}_{N}^{0}\left[a_{k}(0-)\right]},
$$

where $V(0-)=\lim _{t \uparrow 0} V(t)$ and $a_{k}(0-)=\lim _{t \uparrow 0} a_{k}(t)$. The corresponding estimator is given by

$$
Z_{k, m}^{-}(C ; N)=\frac{\sum_{n=1}^{m} 1_{\left\{V\left(T_{n}-\right) \in C\right\}} a_{k}\left(T_{n}-\right)}{\sum_{n=1}^{m} a_{k}\left(T_{n}-\right)} .
$$

\section{IMPLEMENTATION}

Since the traffic is not fluid in practice, we have to consider the practical implementation of the estimator $Z_{k, m}(C ; N)$ in (11) as well as $Z_{k, m}^{-}(C ; N)$ in (12) for $k=1, \ldots, K$ and $C \in \mathfrak{B}\left(\mathbb{R}_{+}\right)$. Note that the network is monitored at the arrival epochs of the active probe packets. When the influence of the active probe packets on the network is negligible, we can assume that the sampling process $N$ and $\left\{\left(V(t), a_{k}(t) ; k=1, \ldots, K\right)\right\}_{t \geq 0}$ are mutually independent. In this case, the estimator $Z_{k, m}(C ; N), C \in \mathfrak{B}\left(\mathbb{R}_{+}\right)$, in (11) can be implemented as either of the following:

$$
\begin{aligned}
& \tilde{Z}_{k, m}^{+}(C ; N)=\frac{\sum_{n=1}^{m} 1_{\left\{V\left(T_{n}\right) \in C\right\}} \tilde{a}_{k}^{+}\left(T_{n}, \delta\right)}{\sum_{n=1}^{m} \tilde{a}_{k}^{+}\left(T_{n}, \delta\right)}, \\
& \tilde{Z}_{k, m}^{-}(C ; N)=\frac{\sum_{n=1}^{m} 1_{\left\{V\left(T_{n}-\right) \in C\right\}} \tilde{a}_{k}^{-}\left(T_{n}, \delta\right)}{\sum_{n=1}^{m} \tilde{a}_{k}^{-}\left(T_{n}, \delta\right)},
\end{aligned}
$$

where $\tilde{a}_{k}^{+}(t, \delta)$ and $\tilde{a}_{k}^{-}(t, \delta)$ denote the numbers of packets transmitted by user $k$ observed during $[t, t+\delta)$ and $[t-\delta, t)$, respectively, and $\delta$ is a small positive number (Fig. 3). In 
TABLE I

TRAFFIC MODEL.

\begin{tabular}{|c|c|c|c|c|c|c|c|c|}
\hline \multirow[b]{2}{*}{$\begin{array}{c}\text { traffic } \\
\text { type }\end{array}$} & \multirow[b]{2}{*}{$\begin{array}{l}\text { connection } \\
\text { ID }\end{array}$} & \multicolumn{2}{|c|}{ transport layer } & \multicolumn{5}{|c|}{ application layer } \\
\hline & & protocol & $\begin{array}{l}\text { packet } \\
\text { length }\end{array}$ & $\begin{array}{c}\text { mean } \\
\text { ON period }\end{array}$ & $\begin{array}{c}\text { mean } \\
\text { OFF period }\end{array}$ & $\begin{array}{l}\text { ON/OFF length } \\
\text { distribution }\end{array}$ & $\begin{array}{c}\text { shape } \\
\text { parameter }\end{array}$ & $\begin{array}{c}\text { rate at } \\
\text { ON period }\end{array}$ \\
\hline type 1 & $\# 1-\# 5$ & $\overline{\mathrm{TCP}}$ & $1.5 \mathrm{~KB}$ & $10 \mathrm{~s}$ & $5 \mathrm{~s}$ & exp & - & $1 \mathrm{Mbps}$ \\
\hline type 2 & $\# 6-\# 10$ & TCP & $1.5 \mathrm{~KB}$ & $5 \mathrm{~s}$ & $10 \mathrm{~s}$ & $\exp$ & - & $1 \mathrm{Mbps}$ \\
\hline type 3 & $\# 11-\# 15$ & TCP & $1.5 \mathrm{~KB}$ & $5 \mathrm{~s}$ & $15 \mathrm{~s}$ & pareto & 1.5 & $1.5 \mathrm{Mbps}$ \\
\hline type 4 & $\# 16-\# 20$ & TCP & $1.5 \mathrm{~KB}$ & $1 \mathrm{~s}$ & $19 \mathrm{~s}$ & pareto & 1.5 & $1.5 \mathrm{Mbps}$ \\
\hline
\end{tabular}

the implementation of (13) [resp. (14)], $\left\{V\left(T_{n}\right)\right\}_{n=1}^{m}$ [resp. $\left.\left\{V\left(T_{n}-\right)\right\}_{n=1}^{m}\right]$ can be obtained by active monitoring of the network and $\left\{\tilde{a}_{k}^{+}\left(T_{n}, \delta\right)\right\}_{n=1}^{m}$ [resp. $\left.\left\{\tilde{a}_{k}^{-}\left(T_{n}, \delta\right)\right\}_{n=1}^{m}\right]$ is by passive monitoring.

Let us consider the following two ways to realize the passive monitoring device in CoMPACT Monitor (see the bottom figure in Fig. 1).

- Realtime counting:

The passive monitoring device performs packet filtering and counting, on-line. It records a sequence of the number of packets observed during $[t, t+\delta)$ or $[t-\delta, t)$, for each $k$.

- Non-realtime counting:

The passive monitoring device monitors all packets and records a part of them, which includes sufficient information for packet filtering. Another procedure extracts packet data observed during $[t, t+\delta)$ or $[t-\delta, t)$ from the recorded packet data, and counts them for each $k$.

In the first case, the implementation of (13) is easier than that of (14). This is because, while the arrival of the active probe packet at $T_{n}, n=1, \ldots, m$, triggers the counting of the user packet arrivals during $\left[T_{n}, T_{n}+\delta\right)$ in the implementation of (13), we have to record all the arrival epochs of user packets to keep ready for the arrivals of active probe packets in that of (14). In addition, since realtime counting requires high-speed packet filtering, it is difficult to realize for a broadband link.

In the second case, both the implementations of (13) and (14) are easy. So, when the influence of the active probe packets on the network is not negligible, by letting the active probe packet stream be a Poisson process (the case of Remark 3), $\tilde{Z}_{k, m}^{-}(C ; N)$ in (14) can be used as the implementation of $Z_{k, m}^{-}(C ; N)$ in (12). In addition, since the passive monitoring device is simple, it can be applicable to a broadband link.

\section{CHARACTERISTICS OF COMPACT MONitor: SIMULATION RESULTS}

\section{A. Network and Traffic Models}

We investigate the characteristic of CoMPACT Monitor by simulation. We use the network model shown in Fig. 4, which has 20 pairs of the source/destination hosts. Although the network model looks simple, it can be considered as part of a whole network, extracted as a pair of source and destination subnetworks. Link capacity between hosts and a router is 1.5 Mbps and that between routers is $10 \mathrm{Mbps}$. Each host

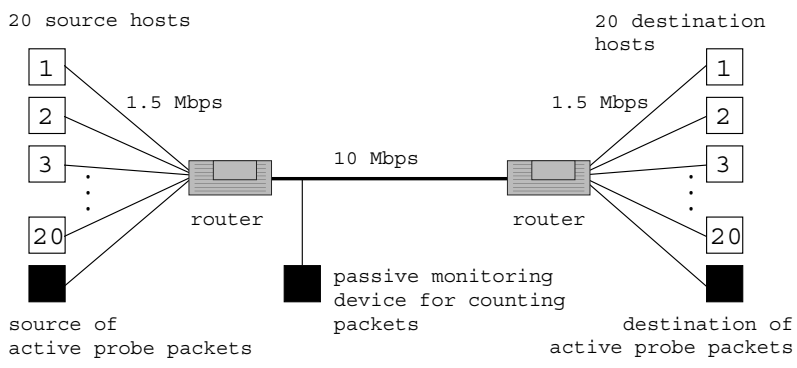

Fig. 4. Network model.

on the left in Fig. 4 is a source and transfers data to the corresponding host on the right. Characteristics of application traffic are ON-OFF processes and are categorized into four different types described in Table I. Each application traffic type is assigned to five hosts and traffic is transferred by TCP/IP. The size of user packets is 1500 bytes.

In addition, to support the use of active probe packets, there is a pair of hosts for sending and receiving the active probe packets. They are connected in the same manner as user hosts. The active probe packets are 64 bytes long and are inserted into the network according to Poisson process.

The passive monitoring device shown in Fig. 4 is for counting packets in order to determine the likelihood ratio.

We conducted a 3600-s simulation using ns2 [4] and monitored the queueing delay distributions for both active probe packets and user packets. Simultaneously, we calculated the queueing delay distribution by using the framework of CoMPACT Monitor. Here, queueing delay means the sum of the waiting time at buffers in the routers on the flow, i.e., endto-end delay is obtained from the sum of the queueing delay and fixed delay determined by link capacity and packet size.

\section{B. Queueing Delay Distributions for Individual Users}

In this subsection, we show that CoMPACT Monitor can estimate the queueing delay distribution for each user from one common sequence of delay measurements using active probe packets.

We have chosen a simpler implementation of CoMPACT Monitor (13). To obtain $\tilde{a}_{k}^{+}\left(T_{n}, \delta\right), n=1,2, \ldots$, the passive monitoring device counts the number of packets. The length of the period $\left[T_{n}, T_{n}+\delta\right)$ for counting packets is $20 \mathrm{~ms}$, i.e., $\delta=20 \mathrm{~ms}$. Hereafter, we call the period $\left[T_{n}, T_{n}+\delta\right)$ the neighborhood of $T_{n}$ and $\delta$ the length of the neighborhood. 


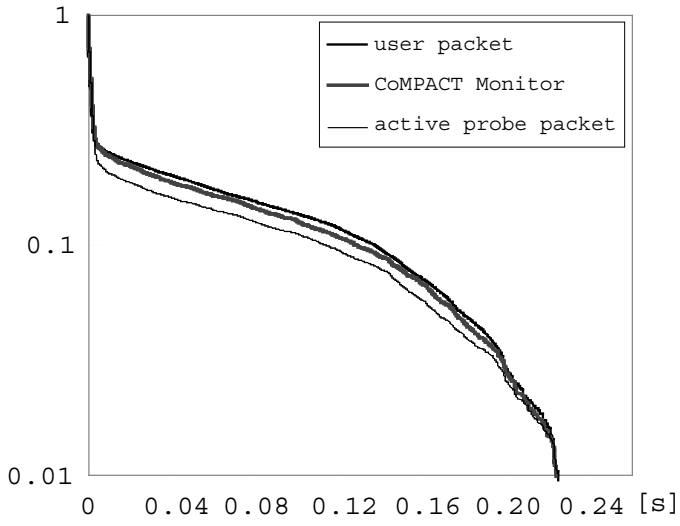

Fig. 5. Queueing delay distributions for connection \#1.

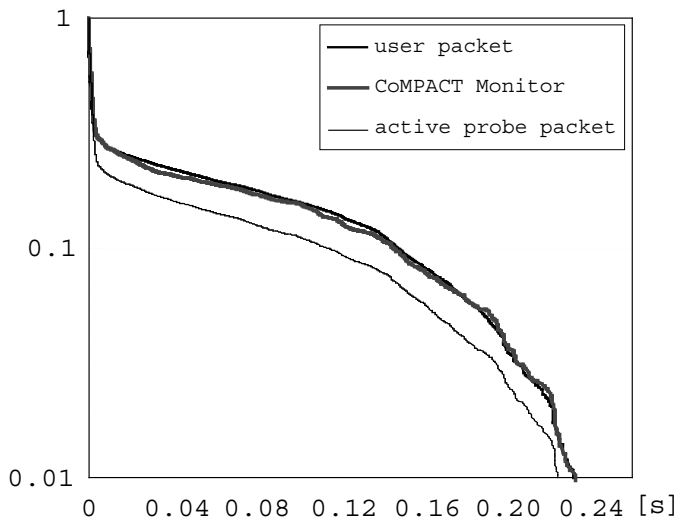

Fig. 6. Queueing delay distributions for connection \#6.

We insert active probe packets into the network to have Poisson arrivals with a mean interval of $500 \mathrm{~ms}$. The extra traffic caused by the active probe packets is only about $0.01 \%$ of the link capacity of $10 \mathrm{Mbps}$ so the influence on user traffic is negligible.

Among the 20 connections shown in Table I, we show the queueing delay distributions (complementary distributions) for connections \#1, \#6, \#11, and \#16, representing four different traffic types, in Figs. 5-8. These figures show the queueing delay distributions of user packets, those of active probe packets, and those obtained from CoMPACT Monitor. The distributions of active probe packets are, of course, the same in these figures.

Although simple active probe packets cannot estimate the queueing delay that users experience, an implementation of CoMPACT Monitor (13) can estimate the queueing delay according to user traffic characteristics.

\section{Implementation and Neighborhood}

This subsection shows the difference between two implementations (13) and (14), and investigates the influence of neighborhood size, $\delta$.

We show a result for connection \#16 among the connections shown in Table I. The traffic type including connection \#16 has the smallest average rate and the strongest burstiness.

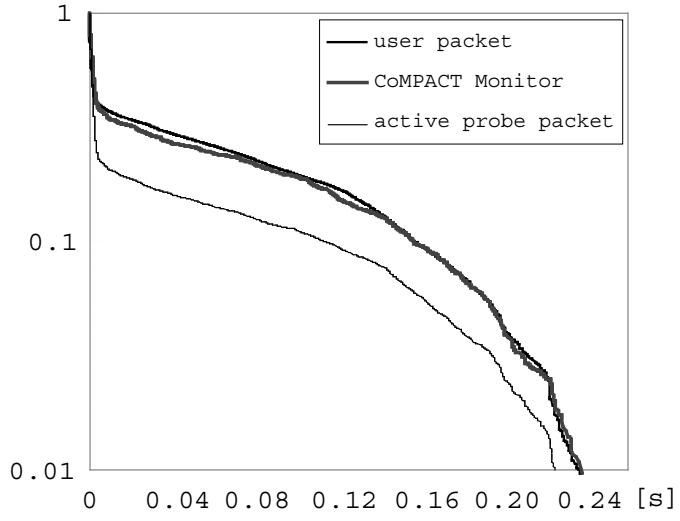

Fig. 7. Queueing delay distributions for connection \#11.

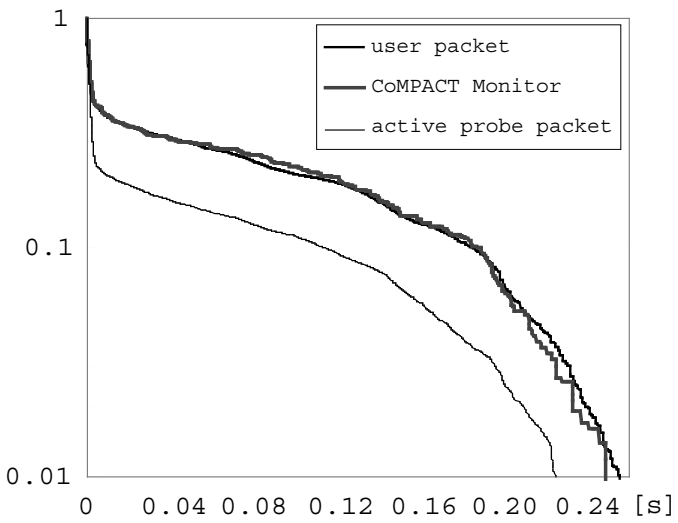

Fig. 8. Queueing delay distributions for connection \#16.

The significant difference between implementations (13) and (14) is in the difference between $\tilde{a}_{k}^{+}\left(T_{n}, \delta\right)$, and $\tilde{a}_{k}^{-}\left(T_{n}, \delta\right), n=1,2, \ldots$. That is, the period for counting packets is after $T_{n},\left[T_{n}, T_{n}+\delta\right)$, or before $T_{n},\left[T_{n}-\delta, T_{n}\right)$ (Fig. 3).

Figure 9 shows queueing delay distributions for both implementations (13) and (14) with $\delta=20 \mathrm{~ms}$. Active probe packets are inserted into the network to have Poisson arrivals with a mean interval of $500 \mathrm{~ms}$. From this figure, both implementations give almost the same distributions. Since implementation (13) is simpler than that of (14), CoMPACT Monitor can be implemented as (13) when the extra traffic for active probe packets is negligible.

Next, we investigate the characteristics of CoMPACT Monitor with respect to $\delta$. Figure 10 shows queueing delay distributions for connection \#16 obtained from the implementation (13) with $\delta=20,40$, and $80 \mathrm{~ms}$. Other conditions are the same as in the above simulation.

This figure shows that the accuracy of implementation (13) is independent of the neighborhood size, at least, in this range of these simulations. This means that it is not necessary to set a very short size for $\delta$, and we can avoid quick switching of the packet counter between start and stop by setting a medium length for $\delta$. 


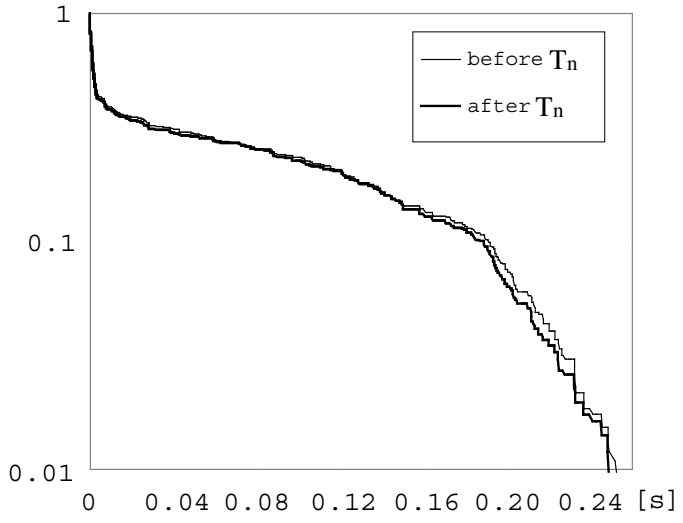

Fig. 9. Queueing delay distributions for two implementations, (13) and (14).

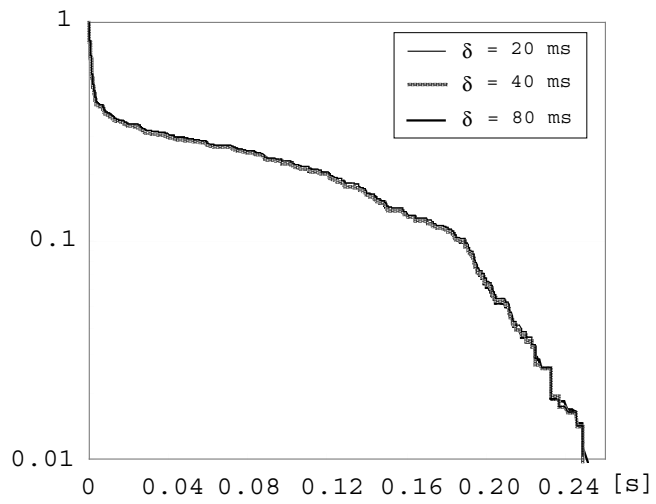

Fig. 10. Queueing delay distributions for different neighborhood sizes.

\section{Interval of Active Probe Packets}

This subsection shows the characteristics of CoMPACT Monitor with respect to the interval of active probe packets.

Similarly to the above simulations, we choose connection \#16. Figures 11 and 12 show queueing delay distributions for connection \#16 obtained by the implementation (13) with $1000 \mathrm{~ms}$ and $250 \mathrm{~ms}$ for the mean active probe packet inter$\mathrm{val}$, respectively. Queueing delay distributions experienced by connection \#16 are also shown in these figures. In both cases, the extra traffic caused by active probe packets is negligible, about $0.005 \%$ and $0.02 \%$ of the link capacity, respectively.

We compare them with Fig. 8 for a mean interval of $500 \mathrm{~ms}$. We can recognize that a smaller interval gives a more accurate delay estimation. Because the interval of active probe packets does not influence the accuracy of estimation from the framework of CoMPACT Monitor, the interval is not essential but the number of samples is. To verify this, we compare relative errors

\section{$\frac{\mid(x \% \text { ile from CoMPACT Monitor })-(x \% \text { ile of user delay }) \mid}{(x \% \text { ile of user delay })}$}

for the 90\%ile, 95\%ile, and 99\%ile of the distributions for three different mean intervals, and each is shown in Figs. 1315. They are obtained from five independent simulations. The relative errors of $90 \%$ ile value are almost the same but that of 99\%ile value is different. These results imply that we cannot

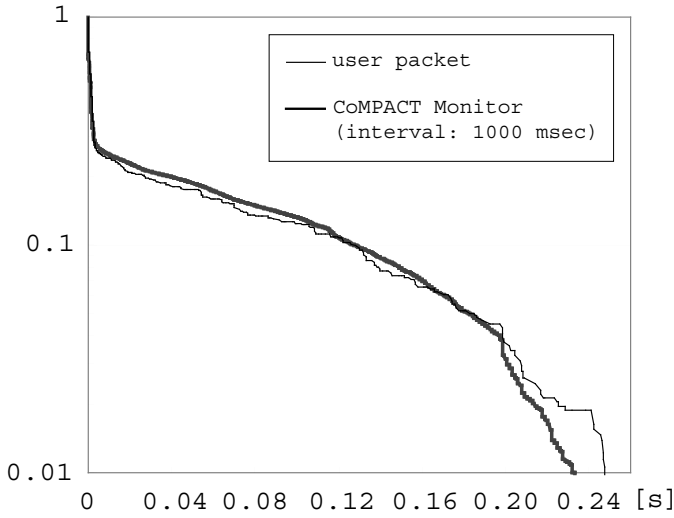

Fig. 11. Queueing delay distribution for interval of active probe packets: $1000 \mathrm{~ms}$.

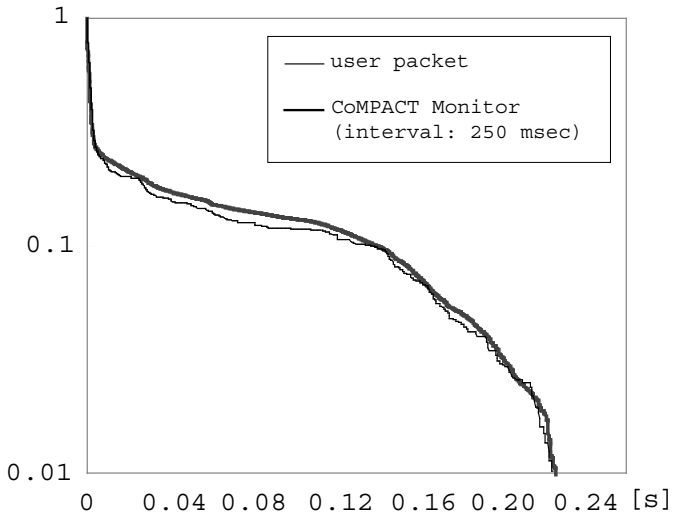

Fig. 12. Queueing delay distribution for interval of active probe packets: $250 \mathrm{~ms}$.

have enough samples to estimate the 99\%ile value for a 1000 ms interval of active probe packets.

From the above discussion, it is necessary to determine an appropriate interval for active probe packet taking into consideration the following factor:

- Required accuracy of estimation,

- Time constraint to finish the monitoring, and

- Permissible extra traffic caused by active probe packets.

\section{CONCLUSIONS}

In this paper, we showed the mathematical formulation of CoMPACT Monitor without assuming stationarity or ergodicity for user traffic. The characteristics and performance associated with the implementation issues in CoMPACT Monitor were investigated by simulation. Unlike conventional active measurements, CoMPACT Monitor can estimate the delay distribution for individual flows that we want to measure. In addition, it enables us to make a scalable and lightweight measurement system and can be implemented in a simple way.

Since the passive monitoring device described in this paper counts the number of packet, we can obtain the delay distribution with respect to packet. In the framework of CoMPACT Monitor, if the passive monitoring device accumulates the 


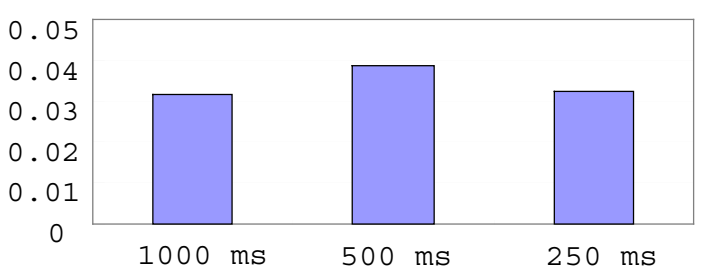

Fig. 13. Relative error of $90 \%$ ile value of queueing delay.

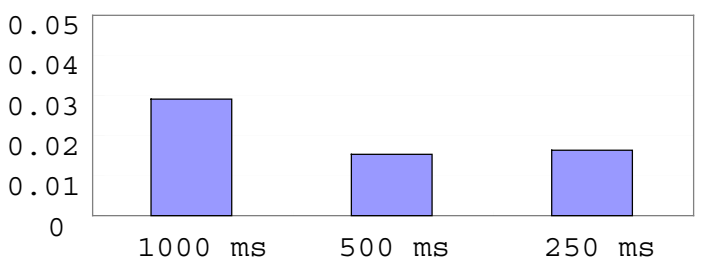

Fig. 14. Relative error of $95 \%$ ile value of queueing delay.

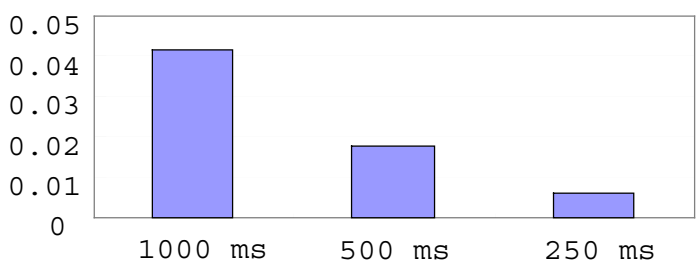

Fig. 15. Relative error of $99 \%$ ile value of queueing delay.

bytes of packets, we can obtain the delay distribution with respect to byte.

We focused on one-way delay in this paper. Although this is important for video streaming, VoIP, and the other realtime services, there are other significant QoS parameters: for example, loss, round-trip delay, throughput, and Web server workload experienced by a user packet flow. We should extend CoMPACT Monitor for other QoS parameters. These issues are for further study.

\section{REFERENCES}

[1] CAIDA cooporative association for internet data analysis. http: //www. caida.org/tools/.

[2] NLANR measurement and operation analysis team. http://moat.nlanr.net/.
[3] IPMA: Internet performance measurement and analysis. http://www.merit.edu/ipma/.

[4] UCB/LBNL/VINT Network Simulator - ns (version 2). http://www.isi.edu/nsnam/ns/.

[5] M. Aida, K. Ishibashi and T. Kanazawa. CoMPACT-Monitor: Changeof-measure based passive/active monitoring - weighted active sampling scheme to infer QoS-. Proc. of IEEE SAINT 2002 Workshop (2002) $119-125$.

[6] G. Almes, S. Kalidindi, and M. Zekauskas. A one-way delay metric for IPPM. RFC2679, (1999).

[7] G. Almes, S. Kalidindi, and M. Zekauskas. A one-way packet loss metric for IPPM. RFC2680, (1999).

[8] S. Asmussen. Applied Probability and Queues. Wiley (1987).

[9] F. Baccelli and P. Brémaud. Elements of Queueing Theory: Palmmartingale Calculus and Stochastic Recurrences. Springer (1994).

[10] J.C. Bolot. Characterizing end-to-end packet delay and loss in the internet. Journal of High Speed Networks, 2(3), September (1993) 289_ 298.

[11] P. Brémaud. Markov Chains: Gibbs Fields, Monte Carlo Simulation, and Queues. Springer (1999)

[12] R. Caceres, N.G Duffield, J. Horowitz, D.F. Towsley, and T. Bu. Multicast-based inference of network-internal characteristics: Accuracy of packet loss estimation. Proc. of IEEE INFOCOM'99, (1999) 371-379.

[13] D.J. Daley and D. Vere-Jones. An Introduction to the Theory of Point Processes. Springer (1988).

[14] P. Glynn and K. Sigman. Independent sampling of a stochastic process. Stochastic Process. Appl., 74 (1998) 151-164.

[15] K. Ishibashi, T. Kanazawa, and M. Aida. Active/passive combinationtype performance measurement method using change-of-measure framework. Proc. of IEEE GLOBECOM 2002, (2002).

[16] S. Keshave. A control theoretic approach to flow control. Proc. of ACM SIGCOM'91, (1991) 3-15.

[17] T. Lindh. A framework for embedded monitoring of QoS parameters in IP-based virtual private networks. Proc. of Passive and Active Measurements (PAM 2001), 2001.

[18] T. Lindh. A new approach to performance monitoring in IP networks - combining active and passive methods. Proc. of Passive and Active Measurements (PAM 2002), 2002.

[19] V. Paxson and S. Floyd. Wide-area traffic: The failure of Poisson modeling. IEEE/ACM Trans. on Networking, 3(3), June (1995) 226244.

[20] V. Paxson, J. Mahdavi, A. Adams, and M. Mathis. An architecture for large-scale Internet. IEEE Communications, 36(8) (1998) 48-54.

[21] V. Paxson. End-end Internet packet dynamics, IEEE/ACM Trans. on Networking, 7(3) (1999) 277-292.

[22] V. Paxson, G. Almes, J. Mahdavi, and M. Mathis. Framework for IP performance metric. RFC 2330, (1998).

[23] S. Savage, A. Collins, E. Hoffman, J. Snell, and T. Anderson. The endto-end effects on Internet path selection. Proc. of ACM SIGCOM'99, (1999) 289-299

[24] W.R. Stevens. TCP/IP illustrated, Vol. 1. Addison-Wesley, Reading MA, 1994.

[25] R.W. Wolff. Poisson arrivals see time averages. Oper. Res., 30 (1982) 223-231. 